\title{
Ueber eine eigenthümliche Form von Functionen einer complexen Variabeln und über transcendente Gleichungen, die keine Wurzeln haben.
}

(Vorgetragen in der Sitzung der math.-phys. Klasse der K. Bayr. Akademie d. W. am 6. März 1869.)

(Von Herrn Ludwig Seidel in München.)

1.

Im Folgenden bezeichnet einfach

$\lim \boldsymbol{G}$

die Grenze, welcher sich eine von $n$ 'abhängige Grösse $G_{n}$ dann nähert, wenn das reell und positiv gedachte $n$ über alle Grenzen wächst. Die Voraussetzung der Existenz einer solchen Grenze schliesst in sich die andere, dass es gleichgültig ist, ob $n$ continuirlich oder discontinuirlich, z. B. durch ganze Zahlen fortschrejtend, ohne Ende wächst; man kann daher $\lim G$ nach Belieben sofort durch eine der gewöhnlichen unendlichen Formen darstellen, z. B. als Reihe

oder als Integral

$$
\lim \boldsymbol{G}=\boldsymbol{G}_{0}+\left(\boldsymbol{G}_{\mathbf{1}}-\boldsymbol{G}_{0}\right)+\left(\boldsymbol{G}_{2}-\boldsymbol{G}_{\mathbf{1}}\right)+\cdots
$$

$$
\lim G=G_{a}+\int_{a}^{\infty} \frac{\partial G_{n}}{\partial n} d n
$$

(vorausgesetzt, dass $\frac{\partial \boldsymbol{G}_{n}}{\partial \boldsymbol{n}}$ zwischen den positiven Grenzen $a$ und $\infty$ eine continuirliche Function von $n$ bleibt) u. s. w.; man könnte sonach im Folgenden, wo wir uns an die zuerst aufgestellte Schreibweise halten, dieselbe nach Belieben durch eine der anderen Formen ersetzen. Enthält $G$ neben dem reellen $n$ noch eine als complex angenommene Variable $x=u+v i=r e^{\omega i}$, so wird übrigens $\lim G$ im Allgemeinen als eine transcendente Function von $x$ anzusehen sein, auch wenn $G_{n}$ diese Grösse nur algebraisch enthält.

2.

Nimmt man

$$
\text { (I.) } \quad G_{n}=\frac{n}{n+x^{n}}
$$


so ist $\lim G=1$ für alle $x$, dẻren Modul (,absoluter Werth"6) $r \leqq 1$ ist, dagegen gleich Null für alle $x$, deren $r>1^{*}$ ). Wenn man also für die Unbekannte $x$ die Gleichung statuirt, nach welcher $\lim G$ irgend etwas anderes als Null oder Eins sein soll, so hat dieselbe gar keine Wurzel der complexen Form $u+v i$, obgleich der Ausdruck $\lim G$ für alle $x$ dieser Form endlich bestimmt ist. Ich weiss nicht, ob man auf die Existenz derartiger transcendenter Gleichungen, welche sich der Analogie der algebraischen sowie derjenigen zahlreicher anderer transcendenter Gleichungen entziehen, schon geachtet hat. In unserem Beispiele könnte das spurlose Verschwinden aller Wurzeln für $n=\infty$ auf den ersten Anblick auffallen, da für jedes endliche $n$ die Gleichung $n$ Wurzeln hat: die Grenzen der Wurzeln der Gleichung $G_{n}=a$ sind hier nicht Wurzeln der Grenzgleichung $\lim G=a$, was sich durch den Umstand erklärt, dass sie im letzteren Ausdrucke auf unendlich hoher Potenz erscheinen. Wollte man übrigens $\lim G$ nicht als eine echte Function von $x$ anerkennen, weil jene Grösse sich im Allgemeinen als Constante verhält und nur an der Peripherie des Kreises $r=1$ springt, so liesse sich sofort etwa

$$
\psi \boldsymbol{x}+(\varphi x-\psi \boldsymbol{x}) \lim \boldsymbol{G}
$$

an ihre Stelle setzen: dieser Ausdruck ist $\varphi x$ für $r \leqq 1$ und $\psi x$ für $r>1$, er kann also keinem Werthe gleich gemacht werden, der so beschaffen ist, dass $\varphi x$ für kein $r \leqq 1$ und $\psi x$ für kein $r>1$ ihn annimmt. Die durch den neuen Ausdruck repräsentirte, im Allgemeinen mit $x$ überall veränderliche Function wird noch, wenn $\varphi x$ und $\psi x$ Functionen gewöhnlicher Art sind, bei $r=1$ discontinuirlich sein, weil für solche Functionen nicht allgemein $\varphi\left(1 . e^{\omega i}\right)=\psi\left(1 . e^{\omega i}\right)$ sein kann, ohne dass überhaupt $\varphi\left(r e^{\omega i}\right)=\psi\left(r e^{\omega i}\right)$ wird; aus dem weiter Folgenden wird man indess entnehmen, dass auch Ausdrücke für durchaus continuirliche Functionen sich bilden lassen, die für keinen complexen Werth von $x$ versagen, und doch gewisse, z. B. negative Werthe gar nie annehmen können.

Da, wo in der elementaren Mathematik die Frage nach den Quadratwurzeln negativer Zahlen zuerst auftritt, überzeugt man sich zwar, dass dieselben in dem vorher aufgestellten Gebiete der positiven und negativen Zahlen sich nicht finden, aber nicht, dass es ein Unsinn ist, sie zu postuliren. Ihre Definition durch dieses Postulat und ihre Einführung in die Rechnung hat sich

*) Der einfachere Ausdruck, bei welchem im Zähler und im ersten Gliede des Nenners 1 statt $n$ gesetzt ist, wird hier vermieden wegen der Unbestimmtheit seines imaginären Bestandtheiles für $r=1$. 
denn auch als statthaft und sehr erfolgreich erwiesen. Nachdem sich nun zeigt, dass transcendente Gleichungen sich aufstellen lassen, die auch in dem erweiterten Felde des Complexen keine Wurzeln finden, so könnte man sich wohl denken, dass irgend eine Gleichung solcher Art zur Definition eines noch fremden Gebietes von Imaginären benutzt werden könnte, - obgleich bei der nothwendigen Complication der Definitionsgleichung von der Einführung solcher Formen in die Rechnung schwerlich ein umfassender Erfolg sich erwarten liesse. Gewiss ist aber, dass die besondere Transcendente, welche uns hier Gleichungen ohne complexe Wurzeln geliefert hat, zur Definition neuer Imaginären nicht dienen könnte, weil hier die Unmöglichkeit, die betreffende Gleichung zu erfüllen, Folge eines inneren Widerspruches derselben mit sich selbst ist, der deshalb hier vorkommen kann, weil eine Grenzgleichung $\lim G=a$ eigentlich mehrere (unendlich viele) Einzelgleichungen, geltend für die verschiedenen unendlich grossen Zahlen $n$, in sich enthält. Sollte nehmlich für einen Werth $Z$, der weder Null noch Unendlich ist, werden

$$
\lim \frac{n}{n+x^{n}}=\frac{1}{1+Z},
$$

so müsste man für ein unendliches $n$ mit gleichem Rechte haben

$$
\frac{x^{n}}{n}=Z \quad \text { und } \quad \frac{x^{n+1}}{n+1}=Z \text {; }
$$

hieraus $x \frac{n}{n+1}=1$, d. h. $x=1$, - und da dieser aus dem Postulate nothwendig sich ergebende Werth demselben gleichwohl nicht entspricht, so ist die gestellte Forderung an sich unerfüllbar.

3.

Schreibt man $\frac{x}{\vartheta}$ statt $x$ in unserem Ausdrucke $G$, wobei $\vartheta$ eine reelle positive Grösse vorstellen soll, und nimmt man die Ergänzung zu 1, so erhält man

$$
\text { (II.) } \quad H_{n}=\frac{x^{n}}{x^{n}+n \vartheta^{n}},
$$

und man hat $\lim \boldsymbol{H}=1$, sofern $\vartheta$ kleiner ist, als der Modul $r$ von $x$, dagegen ist $\lim \boldsymbol{H}=\mathbf{0}$, sowie $\boldsymbol{\vartheta}$ den Werth $\boldsymbol{r}$ erreicht oder überschreitet. Die Grösse $\lim \boldsymbol{H}$ kann nun nach Art des Dirichletschen Discontinuitäts-Factors benutzt werden; multiplicirt man sie mit $\frac{\partial f(\vartheta)}{\partial \vartheta}$, welcher Ausdruck eine continuirliche 
Function von $\vartheta$ sein soll, und integrirt man nach 9 von Null bis $+\infty$, so hat das Element des Integrals nur so lange nicht verschwindende Werthe, als $\vartheta<r$; man erhält daher

$$
\text { (III.) } \int_{0}^{\infty} \lim \frac{x^{n}}{x^{n}+n \vartheta^{n}} \frac{\partial f(\vartheta)}{\partial \vartheta} d \vartheta=f(r)-f(0),
$$

sodass eine beliebige Function des Moduls $r$ von $x$ als Function von $x$ selbst dargestellt ist. Nimmt man z. B. $f(\boldsymbol{\vartheta})=\boldsymbol{\vartheta}$, so ergiebt sich

$$
\text { (IV.) } \quad r=\bmod x=\int_{0}^{\infty} \lim \frac{x^{n}}{x^{n}+n \vartheta^{n}} d \vartheta .
$$

In gleicher Weise erhält man $r^{2}=u^{2}+v^{2}$, indem man setzt $f(\vartheta)=\vartheta^{2}$, und hiermit findet sich auch zu

der conjugirte Werth

$$
x=u+v i
$$

$$
\text { (V.) } u-v i=2 \int_{0}^{\infty} \lim \frac{x^{n-1} \vartheta}{x^{n}+n \vartheta^{n}} d \vartheta \text {. }
$$

Es ist klar, dass nun auch die beiden Bestandtheile von $x, u$ und $v$, sich einzeln als Functionen von $x$ sofort darstellen lassen, - und hiermit denn weiter, dass mit Hilfe solcher Ausdrücke, wie die hier angewandten, jede beliebige Function zweier Variabeln $u$ und $v$ als Function der Einen complexen Variabeln $x=u+v i$ sich ausdrücken lässt.

Bei der Einschränkung des Begriffes der Function einer complexen Variabeln auf dasjenige engere Gebiet, in welchem sich dieser Begriff bereits so fruchtbar erwiesen hat, d. h. bei der Beschränkung auf solche Functionen $F x$, welche einen von der ,Richtung ${ }^{66}$ der Variation von $x$ unabhängigen Differentialquotienten $\frac{d F x}{d x}$ haben, erscheint bekanntlich weder der Modul von $x$, noch der conjugirte Werth, noch Eine der beiden Componenten $u$, $v$ als eine Function von $x$. Die Kraft mathematischer Ausdrücke, in der Darstellung von Functionen reicht, wie man sieht, weit über das Gebiet jenes engeren Begriffes derselben hinaus. Eine Klasse von Formen, welche in gewisser Beziehung etwas Unfügsames haben, scheint gerade hier eine Stelle zu finden, an welcher sie sich nicht ersetzen lassen; denn wenn $F x$ keinen von der Richtung der Variation von $x$ unabhängigen oder durch $x$ allein ausdrückbaren Differentialquotienten hat, wie dies z. B. bei dem Modul von $x$ der Fall ist, so muss jeder mathematische Ausdruck von $F x$ durch $x$ so beschaffen sein, dass er die Differentiation nach $x$ ausschliesst. 
Es sei noch bemerkt, dass man mit Hilfe solcher Ausdrücke wie (IV.) nun auch leicht solche überall continuirliche Functionen zusammensetzen kann, die nicht mehr constant bleiben, während $x$ gewisse Linien und Flächenstücke durchläuft, und deren Werthe gleichwohl die Ebene nur mit Ausschluss gewisser umgrenzter Flächenstücke bedecken. Bezeichnet zum Beispiel $a$ irgend eine positive Grösse, und $r$ den in (IV.) gefundenen Ausdruck von $\bmod x$, so wird, während $x$ alle complexen Werthe annimmt, die Function $x+\frac{a}{r} x$ ihrerseits alle denkbaren Werthe erhalten mit Ausnahme solcher, deren Modul kleiner ist als $a$, d. h. exclusive derjenigen, die einem Kreise zufallen, welcher mit dem Radius $a$ um den Anfangspunkt beschrieben wird. Will man das Integral $r$ im Nenner vermeiden, so wird dasselbe auch geleistet durch die conjugirte Function der v̀origen, nehmlich durch $\frac{r^{2}}{x}+a \frac{r}{x},-$ wo für $r^{2}$ das einfache Integral zu setzen ist, welches man, wie bei (IV.) angezeigt, für diese Grösse erhält.

\section{4.}

Zu der Formel (III.) lässt sich noch zeigen, dass es erlaubt ist, die Ordnung der beiden durch lim. und durch $\int$ bezeichneten Uebergänge umzukehren, indem zum Beispiel das $\int_{0}^{\infty} H_{n} d \vartheta$ bei wachsendem $n$ zu seiner Grenze den Werth $\int_{0}^{\infty} \lim H_{n} d \vartheta$ hat, - obgleich es in der Nachbarschaft von $\vartheta=r V^{n} \frac{1}{n}$ ein (bei zunehmendem $n$ an Breite abnehmendes) Intervall giebt, in welchem sich $\boldsymbol{H}_{n}$ von $\lim \boldsymbol{H}_{n}$ sehr stark unterscheidet. Nur darf nicht, wenn die Veränderung erlaubt sein soll, $\boldsymbol{H}_{n}$ bei jenem Werthe von $\vartheta$ unendlich werden, weil sonst sein Integral aufhört einen endlichen nachweisbaren Werth zu haben; man muss daher, wenn der Richtungscoefficient von $x$ eine Wurzel aus -1 ist, diejenigen Werthe von $n$ überspringen, welche $x^{n}$ zu einer negativen reellen Grösse machen. - Den ausführlichen Beweis, welcher durch Einschaltung geeigneter Zwischengrenzen in dem nach $\boldsymbol{\vartheta}$ genommenen bestimmten Integrale sich führen lässt *), übergehe ich hier um so mehr, da man z. B. die

*) In dem Beweise, welchen ich mir aufgesetzt, habe ich das Integral in fünf einzelne zerlegt, und die inneren Grenzen der beiden äussersten so bestimmt, dass in diesen $\boldsymbol{H}_{n}$ mit $\lim \boldsymbol{H}$ sehr nahe übereinstimmt, - während die Begrenzung des 
Gleichung (IV.) in ihrer neuen Form

$$
r=\bmod x=\lim \int_{0}^{\infty} \frac{x^{n} d \vartheta}{x^{n}+n \vartheta^{n}}=\lim \int_{0}^{\infty} \frac{x^{n} d \vartheta}{x^{n}+\vartheta^{n}}
$$

für eine ganze Zahl $n$ durch wirkliche Herstellung des Integrals leicht verificiren kann. Es ist nicht ohne Interesse, sich davon näher Rechenschaft zu geben, wie es zugeht, dass dies Integral bei unendlich wachsendem $n$ von dem Richtungscoefficienten der Grösse $x$ unabhängig wird. Von den zweierlei Bestandtheilen, welche die Integrale der einzelnen Partialbrüche, in die complexe Form gebracht, enthalten, heben sich nehmlich die logarithmischen Terme in der Gesammtsumme und nach Substitution der Integrationsgrenzen auf; die Glieder mit den Kreisbogen aber erfordern beim Uebergang zu den Grenzen wegen ihrer vieldeutigen Form eine gewisse Vorsicht. Da der Integrationsweg für $\vartheta$, als reelle Grösse, vorgeschrieben ist, so sind die bestimmten Integrale der einzelnen Partialbrüche frei von jeder Mehrdeutigkeit; man muss für jeden Bogen besonders über das Intervall des Anfangs- und Endwerthes so verfügen, dass von jenem bis zu diesem der Arcus sich ohne Discontinuität ändert, während $\vartheta$ die positiven Werthe durchläuft. (Der Fall, in welchem einer der Partialbrüche selbst durch Unendlich geht, ist der von der Betrachtung vorhin ausgeschlossene, für welchen die Umformung der Gleichung (III.) nicht erlaubt ist.) Wenn man, wie schon Anfangs, setzt $x=r e^{\omega i}$, so wird bei der zuletzt aufgeführten Form des Integrales der Nenner irgend eines der Partialbrüche sein

$$
\vartheta-r e^{\omega i+\frac{2 t+1}{n} \pi i}
$$

wobei $t$ eine der ganzen Zahlen von 0 bis $n-1$ vorstellt; ist nun etwa $\omega$ zwischen 0 (inclusive) und $2 \pi$ (exclusive) genommen worden, so wird bei gehöriger Beachtung der eben bezeichneten Vorsicht im bestimmten Integral der Ausdruck für den Bogen ein anderer in den Gliedern, in welchen $\omega+\frac{2 t+1}{n} \pi$ den Werth $2 \pi$ überschreitet, als in den Anfangsgliedern von kleinerem $t$. Bezeichnet man mit $\tau-1$ den grössten Werth von $t$, für welchen noch $\omega+\frac{2 t+1}{n} \pi<2 \pi$ ist, so findet man alsdann durch Summation

mittelsten um $\vartheta=r \sqrt{\frac{1}{n}}$ gelegenen Theiles so eng gewählt wurde, dass in ihm $H_{n}$ mit einer Function von linearem Nenner sich vertauschen liess: 


$$
\int_{0}^{\infty} \frac{x^{n} d \vartheta}{x^{n}+\vartheta^{n}}=\frac{r \pi}{n \sin \frac{\pi}{n}} e^{\omega i+\frac{2 z \pi}{n} i}
$$

Diese Gl eichung lässt sich auch so schreiben:

$$
\int_{0}^{\infty} \frac{x^{n} d \vartheta}{x^{n}+\vartheta^{n}}=\frac{r \pi}{n \sin \frac{\pi}{n}} e^{\Omega_{n} i},
$$

wenn man mit $n \Omega_{n}$ den (positiven oder negativen) Rest bezeichnet, welcher sich ergiebt, wenn man von nw das zunächst gelegene ganze Vielfache von $2 \pi$ abzieht ${ }^{*}$ ); oder noch einfacher so:

$$
\int_{0}^{\infty} \frac{x^{n} d \vartheta}{x^{n}+\vartheta^{n}}=\frac{\pi}{n \sin \frac{\pi}{n}} \sqrt[n]{ } x^{n}
$$

wenn man erklärt, dass unter den $n$ Werthen der Wurzel derjenige genommen werden soll, dessen reeller Bestandtheil möglichst gross ist im algebraischen Sinne. - Man ersieht hieraus, dass das Integral, wie auch $x$ beschaffen sei, nur solche Werthe annimmt, deren Richtungen zwischen $\pm \frac{\pi}{n}$ liegen, indem es immer wieder auf die ersten Werthe zurückspringt, so oft $x$ sich mit $e^{\frac{2 \pi i}{n}}$ multiplicirt. In Folge dieses discontinuirlichen Ganges bedecken die Werthe des Integrales schon für endliche ganze n, soweit sie bestimmt und nachweisbar sind, von der ganzen Ebene nur den Sector zwischen den Richtungen $\pm \frac{\pi}{n}$, welchen sie $n$ mal im Sinne der Drehung von $x$ durchlaufen, während dieses Einmal den ganzen Kreis durchläuft. Andrerseits ist zwar für die Werthe $x$, welche in eine der Richtungen $\frac{\pi}{n} \pm \frac{2 r \pi}{n}$ fallen, der Werth des Integrales nicht definirt und kann nicht schlechthin unendlich gesetzt werden, da er unendliche Elemente von beiderlei Vorzeichen in sich schliesst; jedenfalls aber kann er nur reell gedacht werden, da für solche $x$ der Ausdruck gar nichts. Imaginäres enthält. Für unsere Anwendung kommen die $x$ der bezeichneten Form deshalb nicht in Betracht, weil, wie oben angeführt, bei gegebenem Werthe von $x$ solche $n$ immer übersprungen werden müssen, welche $x^{n}$ negativ reell machen würden: die Grenze des Integrales, für $n=\infty$, reducirt sich also auf den blossen Modul $r$ von $x$ in der Weise, dass der Spielraum, in welchem der Richtungscoefficient $e^{\Omega_{n} i}$ variiren konnte, zuletzt unendlich schmal wird. -

*) Der Fall, in welchem diese Erklärung zweideutig wird, ist der ausgeschlossene. 
5.

Man kann durch Formen, die unseren $\lim G, \lim H$ analog sind, noch mancherlei Functionen besonderer Art darstellen. Als Beispiel sei nur noch. angeführt der Ausdruck

$$
\lim \frac{n f x}{x^{n}+x^{-n}+n}
$$

der (wenn $f x$ nicht unendlich wird) nicht verschwindende Werthe nur annimmt für diejenigen $x$, deren Modul gleich 1 ist, die also zu Punkten auf der Peripherie eines gewissen Kreises gehören. Es ist einleuchtend, dass man ähnlich auch solche discontinuirliche Ausdrücke bilden könnte, welche nur längs einer anderen vorgegebenen Linie nicht Null sind, und auf dieser eine gegebene Function repräsentiren.

Ganz verwandter Natur ist die Darstellung der dem absoluten Werthe nach grössten unter mehreren Grössen $a, b, c \ldots$ durch Formen wie die folgende

oder auch

$$
\lim \frac{a^{n+1}+b^{n+1}+c^{n+1}+\cdots}{a^{n}+b^{n}+c^{n}+\cdots}
$$

$$
\lim ^{n} \sqrt{ }\left\{a^{n}+b^{n}+c^{n}+\cdots\right\}
$$

welche man leicht allgemeiner einrichten kann, um auch die zweitgrösste etc. jener Grössen nach Belieben darzustellen. Diese letzten Formen mögen schliesslich noch an den Nutzen erinnern, der eigenthümlicher Weise gerade auf dem Gebiete der angewandten Mathemàtik aus der Einführung von Grenzausdrücken solcher Art schon für die Wissenschaft resultirt hat: Laplace hat von solchen (Buch II. Cap. 4. der Théorie des probabilités) eine Anwendung gemacht für die Lösung der Aufgabe, Beobachtungsresultate so auszugleichen, dass der grōsste übrig bleibende Fehler möglichst klein wird, und Gräffe hat sie mit vorzüglichem Erfolge für die Auflösung numerischer algebraischer Gleichungen verwerthet.

München, im März 1869. 